\title{
Self-Efficacy Is a Modifiable Factor Associated with Frailty in Those with Minor Stroke: Secondary Analysis of $\mathbf{2 0 0}$ Cohort Respondents
}

\author{
Abodunrin Quadri Aminu ${ }^{a}$ Roderick Wondergem ${ }^{b, c, d}$ Yvonne Van Zaalen ${ }^{b}$ \\ Martijn Pisters ${ }^{b, c, d}$ \\ ${ }^{a}$ School of Nursing, Midwifery and Paramedic Practice, Robert Gordon University, Aberdeen, UK; ${ }^{\circ}$ Department of \\ Health Innovations and Technology, Fontys University of Applied Sciences, Eindhoven, The Netherlands; \\ 'The Center for Physical Therapy Research and Innovation in Primary Care, Julius Health Care Centers, Utrecht, \\ The Netherlands; ${ }^{d}$ Department of Rehabilitation, Physical Therapy Science and Sport, Brain Center, University \\ Utrecht, University Medical Center Utrecht, Utrecht, The Netherlands
}

\section{Keywords}

Self-efficacy · Frailty · Stroke management · Healthcare

\begin{abstract}
Background: Owing to the improvement in acute care, there has been an increase in the number of people surviving stroke and living with its impairments. Frailty is common in people with stroke and has a significant impact on the prognosis after stroke. To reduce frailty progression, potentially modifiable factors should be identified. Increasing levels of self-efficacy influence both behaviour and physical functioning, and therefore it could be a potential target to prevent frailty. Methods: This is a prospective cohort study that involved the secondary analysis of the RISE data to examine the relationship between self-efficacy and frailty. The RISE study is a longitudinal study that consists of 200 adults aged $18+$ years after their first stroke event. Data were collected from the respondents at 3 weeks, 6 months, 12 months, and 24 months after their discharge from the hospital. Frailty was assessed using the multidimensional frailty index with scores ranging from 0 to 1 , and self-efficacy was assessed using the SESx scale, which was dichotomized as low/moderate or high. Frailty trajectories were examined using the repeated linear model. The generalized estimating equation was used
\end{abstract}

karger@karger.com www.karger.com/cee

Karger!

GOPEN ACCESS
C 2021 The Author(s).

Published by S. Karger AG, Basel

This is an Open Access article licensed under the Creative Commons Attribution-NonCommercial-4.0 International License (CC BY-NC) (http://www.karger.com/Services/OpenAccessLicense), applicable to the online version of the article only. Usage and distribution for commercial purposes requires written permission. to examine the relationship between self-efficacy and frailty at baseline and in the future (6-24 months). The B coefficients were reported at $95 \% \mathrm{Cl}$ before and after adjusting for potential confounders (age, gender, stroke severity, education, and social support). Results: A total of 200 responses were analysed, and the mean age of the respondents was $67.78 \pm 11.53$. Females made up $64 \%$ of the sample, and the mean frailty score at baseline was $0.17 \pm 0.09$. After adjusting for confounders, respondents with low self-efficacy had an approximately $5 \%$ increase in their frailty scores at baseline and in the 24-month follow-up period compared to those with high self-efficacy. Conclusion: The result from this study showed that self-efficacy was significantly associated with frailty after stroke. Our findings suggest that self-efficacy may play a role in frailty progression among stroke survivors.

(c) 2021 The Author(s).

Published by S. Karger AG, Basel

\section{Introduction}

According to the global burden of disease report in 2016, stroke is one of the leading causes of mortality worldwide with an estimated 5.5 million deaths and 116 million disability-adjusted life years [1]. Conversely, 50\% of stroke-related deaths have been linked to poor man- 
agement and thus potentially preventable [2]. Frailty is common in people with stroke and has a significant impact on the prognosis after stroke [3]. Almost 25\% of the people with stroke become frail [3], two times more than healthy peers. Frailty is one of the long-term determinants of survival after stroke, and it is associated with cognitive deficit and decline in activities of daily living [4].

Frailty is a clinical syndrome characterized by an increase in the body's susceptibility to stressors [5]. Currently, there is no universally agreed definition of frailty. It has been demonstrated that the deficit accumulation model, which incorporates all aspects of frailty such as physical, affective, and cognitive, is an adequate representation of frailty [6-8]. Although there is no consensus on the definition of frailty [9], frailty has been linked to an increased number of mortalities among stroke patients [10].

In geriatric research, it is objectified that frailty is dynamic and reversible [11-13]. Owing to the increased risk of frailty among stroke patients, reversing frailty will be potentially beneficial to a substantial part of this population. This means that a proper stroke recovery plan should include assessing and managing frailty among patients. However, to proceed, there is a need to investigate the prevalence of frailty in community-dwelling people with stroke.

To effectively manage frailty among those living with stroke, there is a need to also identify its modifiable risk factors. Home-based intervention with both physical function and behavioural components has shown to have positive effects on reducing frailty [14]. In other populations and stroke populations, it has been found that increasing the level of self-efficacy influences both health behaviour and physical functioning and is linked to better health outcomes [15-17]. Self-efficacy is successfully targeted in interventions for people with stroke and linked to decreased progression of cardiovascular diseases [18]. Recently, it was found that high levels of self-efficacy were associated with lower odds of frailty in older adults with chronic disease [19]. Therefore, self-efficacy could be an important modifiable risk factor to prevent frailty among people living with stroke.

Although the association between frailty and self-efficacy was found in older adults with chronic disease, there is a paucity of the literature on the relationship between self-efficacy and frailty among community-dwelling people with a first-ever stroke. Thus, there is a gap in the knowledge of frailty prevalence and frailty trajectory among community-dwelling individuals living with stroke. Understanding frailty progression among these individuals will be crucial to managing frailty-related adverse outcomes among community-dwelling individuals [4]. In this study, we aimed to examine the frailty trajectory among people with a first-ever stroke in the first two years after discharge from facility-based care to the community and investigate the relationship between being frail and self-efficacy.

\section{Materials and Methods}

This is a prospective cohort study that involves the secondary data analysis of the RISE data (see study design).

\section{Participants and Study Design}

The RISE study is a longitudinal study of 200 individuals with a first-ever stroke discharged from facility-based care to the home setting. Participants were recruited from 4 stroke units in The Netherlands and included between February 2012 and April 2017. Patients were included in the RISE study when they had the first clinical diagnosis of haemorrhagic or ischaemic stroke, were discharged from inpatient care to the home and community setting, were $\geq 18$ years at the time of their stroke, and were independent in activities of daily living before stroke (Barthel Index >18) [20]. After written, informed consent was obtained, the demographic, stroke, and care characteristics were extracted from patients' records. Participants were visited within 3 weeks, 6 months, 1 year, and 2 years after returning home by trained researchers. Before the participant was visited at home, a postal questionnaire was sent to obtain psychological characteristics. Data on cognition, activities, and participation outcomes were obtained, and participants received an accelerometer during the visit to objectify movement behaviour.

\section{Measures}

\section{Outcome Variable}

Frailty

Frailty was assessed in this study using the frailty index (FI) [21]. The FI measures accumulated impairments or deficits in the physical, cognitive, and psychosocial domains of health. In this study, a total of 30 deficits from the RISE data were included in the FI to ensure that the outcome estimates are precise [22]. The FI is comparable across studies, even when different numbers ( $>30$ deficits) or types of deficits are counted. Following previous research [22], the deficits were assigned values between 0 and 1 to generate the frailty score. The frailty index score was calculated by adding up each respondent's total deficits and dividing this by 30 . The output score is expected to range between 0.0 and 1.0, where a higher score indicates greater frailty. 


\section{Independent Variable}

Self-Efficacy

The self-efficacy of participants in this study was assessed using the Self-Efficacy for Symptom Management Scale (SESx) [23]. The SESx is a 13-item scale developed to measure perceived self-efficacy. The SESx items were rated on a 10-point scale $(1=$ not at all confident to $10=$ confident) and summed to a total score of 13-130 (0-57 indicates low self-efficacy, 58-114 for moderate self-efficacy, and 115-130 for high self-efficacy).

\section{Covariates/Confounders}

Stroke Severity

The severity of stroke symptoms was measured with the National Institutes of Health Stroke Scale (range, $0-42$ ). The NIHSS has a good predictive record for recovery after stroke. A previous study has shown that a unit increase in the NIHSS score predicted a poorer outcome among stroke survivors. In this study, the NIHSS was included in the analytical model as a discrete variable.

\section{Social Support}

Social support in the RISE study was determined using the 12-scale social support list (SSL). There are 12 questions in the SSL divided into 3 subscales: social support in problem situations, everyday support, and esteem support. Responses from participants were coded 1-4 (rarely or never, occasionally, regularly, and very often, respectively). The sum of individual scores to the 12 questions for each participant was used to calculate the social support level. The social support scores ranged from 4 to 48 , and higher scores indicated better social support levels. We included social support in our analysis as a discrete variable.

\section{Other Covariates}

Educational level was asked using the Dutch classification system and dichotomized into low (score 1-5, up to completed secondary education) and high (score 6-7, completed secondary professional education, university, or higher). Age was included in the analysis as a continuous variable, and sex was coded male or female.

\section{Data Analysis}

SPSS version 25.0 was used to analyse the data. The data view was structured in the long format for the longitudinal analysis of repeated data. Missing data identified in the RISE dataset were imputed using the multiple imputations in SPSS. It is pertinent to ensure that bias that could result from missing data is avoided. We conducted a missing value analysis and examined the pattern in the missing cases. This is necessary as the imputations method is suggested to be dependent on the pattern of the missing data [24]. For instance, the monotone method is appropriate if the data were not missing at random. In our imputation, the fully conditional specification method (Markov Chain Monte Carlos) was utilized because our data contained missing values at random. The imputation of the data was done in 5 iterations to manage the outputs, and the results of the pooled data are reported in this study.

To determine the frailty trajectory among the participants, we compared the mean differences in the frailty scores across four time points using the repeated general linear model (GLM). The repeated contrast option was used for the "within-subject" tests, and the Bonferroni confidence interval adjustment was used to compare the main effects in the GLM model. The frailty scores at the 4 time points were plotted on a box plot, and the frailty index cutoff points of 0.2 and 0.3 were used to describe the prevalence of prefrailty and frailty in the cohort following previous research [22].

We modelled the relationship between the independent variables and frailty using the generalized linear models and generalized estimating equation (GEE) for the longitudinal analysis. The GEE is a variant of the generalized linear model that can be used for repeated data. The generalized linear model does not require a normally distributed response variable to create a predictive model [25]. This way, we were able to achieve parameter estimates from the model without the normality assumption. At first, we examined the cross-sectional relationship between self-efficacy and frailty by including baseline frailty as a continuous response variable. A future frailty variable was developed to examine the relationship between self-efficacy and the combined frailty outcomes at 6 months, 1 year, and 2 years. The future frailty variable was entered into the model in a continuous form as well. In the adjusted GEE model, the covariates were introduced into the model to examine for confounding effects. Backward elimination was used to remove predictors with a $p$ value above $10 \%$ as done in other studies [26]. For the scale predictors, a 1-unit difference in the predictor at baseline is associated with $\mathrm{B}$ units decreasing or increasing future frailty scores. The B coefficient for the categorical variables is interpreted as the combined average frailty scores over time. The multivariate analysis results are presented as B coefficient at $95 \%$ confidence intervals. 
Fig. 1. Frailty trajectory for respondents in the RISE study.

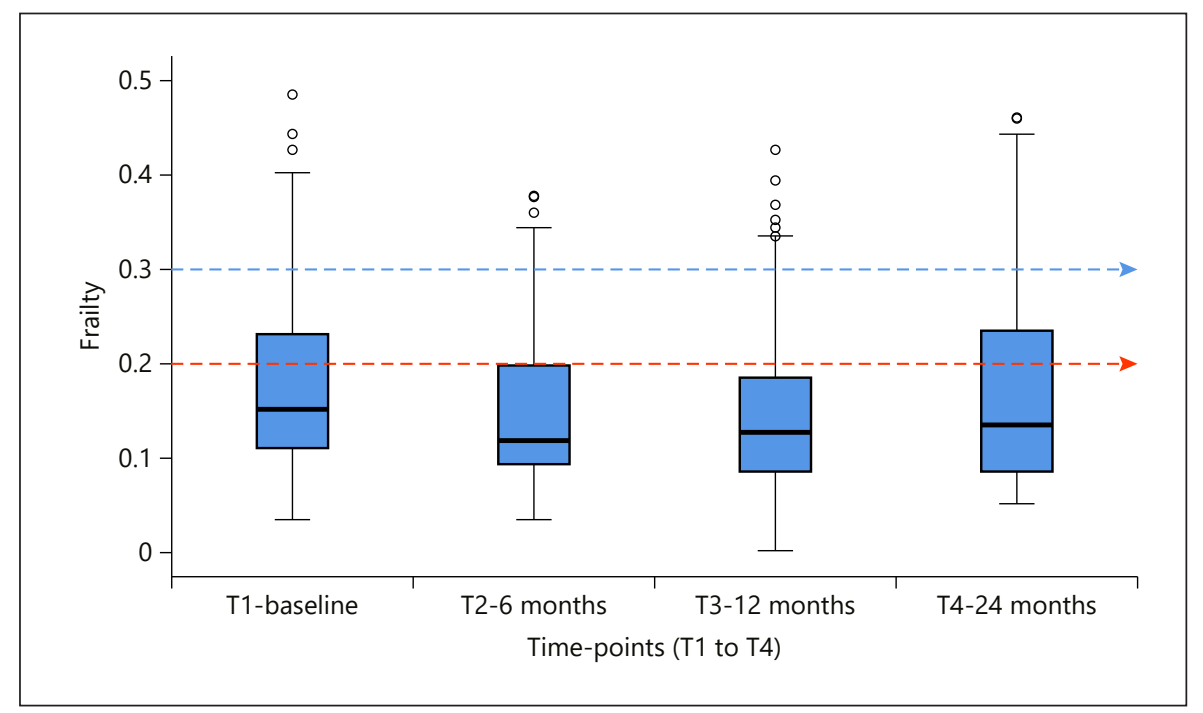

Table 1. Baseline sample characteristics $(n=200)$

\begin{tabular}{ll}
\hline & Total, $N(\%)$ \\
\hline $\begin{array}{l}\text { Age, years (mean } \pm \text { SD) } \\
\text { Gender }\end{array}$ & $67.78 \pm 11.53$ \\
$\quad$ Female & $128(64)$ \\
$\quad$ Male & $72(36)$ \\
Educational status & $140(70)$ \\
$\quad$ Low & $60(30)$ \\
$\quad$ High (post-secondary) & $170(85)$ \\
Self-efficacy & $30(15)$ \\
$\quad$ Low/moderate & $0.17 \pm 0.09$ \\
$\quad$ High & $4.03 \pm 4.02$ \\
Baseline frailty score, mean \pm SD & $30.8 \pm 6$ \\
Stroke severity (NIHSS), mean \pm SD & \\
Social support, mean \pm SD & \\
\hline
\end{tabular}

\section{Results}

A total of 200 responses were analysed in this study. The characteristics of the respondents are presented in Table 1. Of the 200 respondents, $64 \%$ were male and $36 \%$ were female. The overall mean age of respondents was $67.8 \pm 11.5$. The minimum frailty score at baseline was 0.03 , and the maximum was 0.48 . The mean frailty score at baseline was $0.17 \pm 0.09$. A larger percentage $(85 \%)$ of the respondents had low/moderate self-efficacy, and the majority of the respondents (70\%) did not have post-secondary education.

\section{Two-Year Frailty Trajectory among the RISE Cohorts}

The visualization of the frailty progression from baseline to 2-year follow-up can be seen in Figure 1. Please note that the red line and the blue line in Figure 1 indicate the prefrail
Table 2. Mean differences for the frailty scores of the study sample (T1-T4)

\begin{tabular}{lllll}
\hline & T1 frailty & T2 frailty & T3 frailty & T4 frailty \\
\hline Mean scores & \multirow{2}{*}{0.174} & 0.14 & 0.142 & 0.155 \\
T1 & & $-0.034^{*}$ & $-0.032^{*}$ & $-0.019^{*}$ \\
T2 & & 0.002 & $0.016^{*}$ \\
T3 & & & $0.013^{*}$ \\
\hline \multicolumn{2}{r}{$*$ value $<0.05}$. & & \\
\hline
\end{tabular}

cutoff (0.2) and frail cutoff (0.3), respectively. The box plot highlights the average transition of respondents on the frailty scale from baseline to 2 years after hospital discharge. The plot shows that the median frailty score initially reduced for the first 6 months after discharge to the home/community setting but continued to increase up to 2 years. The plot also shows that the prevalence of prefrailty and frailty increased considerably in the cohort 12 months after discharge from the hospital to the home setting.

The mean frailty scores from baseline to 2 years are presented in Table 2. The GLM result showed a significant difference in the future frailty mean scores compared to the baseline at $p<0.01$. There were significant changes in the mean frailty scores between discharge and 6 months and between 1 year and 2 years.

\section{The Relationship between Self-Efficacy and Frailty}

Baseline Frailty

The result of the multivariate analyses using the generalized linear model is presented in Table 3. Self-efficacy 
Table 3. Multivariate analyses using the generalized estimating equation to examine the association between self-efficacy and frailty

\begin{tabular}{|c|c|c|c|c|c|c|}
\hline & $\begin{array}{l}\text { Baseline frailty } \\
\text { (unadjusted) }\end{array}$ & $\begin{array}{l}\text { Baseline frailty } \\
\text { (adjusted) }\end{array}$ & $p$ value & $\begin{array}{l}\text { Future frailty } \\
\text { (unadjusted) }\end{array}$ & $\begin{array}{l}\text { Future frailty } \\
\text { (adjusted) }\end{array}$ & $\begin{array}{l}p \\
\text { value }\end{array}$ \\
\hline Self-efficacy (low) & $0.06(0.04-0.08)$ & $0.05(0.02-0.08)$ & $<0.01$ & $0.04(0.02-0.06)$ & $0.04(0.02-0.06)$ & $<0.01$ \\
\hline Education (below secondary) & & $0.025(0.01-0.05)$ & 0.051 & & $0.02(-0.001$ to 0.04$)$ & 0.09 \\
\hline Age & & $0.001(0-0.002)$ & $<0.05$ & & $0.002(0.001-0.002)$ & $<0.01$ \\
\hline Stroke severity (NIHSS) & & $0.005(0.002-0.008)$ & $<0.01$ & & $0.005(0.002-0.008)$ & $<0.01$ \\
\hline Gender $^{\mathrm{a}}$ (female) & & $0.03(0.01-0.05)$ & $<0.05$ & & - & - \\
\hline
\end{tabular}

Reference group: high self-efficacy, secondary education or above, and male gender. Social support is not included in Table 3 because


$p$ value $>0.1$.

significantly predicted frailty at baseline before and after adjusting for the confounders $(p<0.05)$. Individuals with low/moderate self-efficacy had higher frailty scores compared to individuals with high self-efficacy. The baseline frailty increased by $5 \%$ among individuals with low selfefficacy at baseline.

\section{Future Frailty}

The result of the GEE analysis is also included in Table 3. Self-efficacy remained a significant predictor of future frailty scores of the respondents before and after adjusting for the confounders $(p<0.05)$. The future frailty increased by $4 \%$ among individuals with low self-efficacy at baseline.

\section{Discussion}

Frailty has become a global health concern because of its potentially huge impact on healthcare resources and due to the evidence-practice gap that may exist in the management of frail individuals $[6,27,28]$. Aside from the multidimensional frailty index utilized in this study, the other well-cited definition of frailty is the phenotype model that focuses mainly on physical frailty. The differences and implications of both definitions of frailty have been discussed in previous studies, including the different cutoff points used in the frailty index $[9,29,30]$. Although the multidimensional frailty index tends to produce a higher frailty prevalence compared to the phenotype model, the multidimensional frailty index is better at predicting adverse health outcomes especially among individuals living with chronic conditions [31].

The findings showed that the mean frailty index score for the respondents was 0.17 , and it is consistent with the findings from previous research that analysed the SHARE data to examine frailty prevalence among 11 European countries [32]. The frailty trajectory among the respondents in the present study typifies the pattern of stroke recovery and demonstrates the "golden period" in stroke management [33]. The golden period is the time where the highest impact of rehabilitation on the functional recovery of stroke patients is recorded and usually in the first 3-6 months after stroke [33]. This may explain the reason for the significant drop in the frailty scores of the respondents in this study in the first 6 months after their stroke event.

In this study, we examined the relationship between self-efficacy and frailty after stroke. Self-efficacy has been shown to positively influence the recovery and management of stroke patients [34]. Results from a previous systematic review of 22 studies showed that self-efficacy interventions in stroke management have been linked to positive health outcomes such as quality of life, perceived health status, and physical functioning among stroke survivors [17]. Although there are limited data on the use of self-efficacy intervention to address frailty, our findings suggest that individuals who score low on self-efficacy after stroke may benefit from such interventions. The results of the GEE analysis in this study showed that there was approximately $5 \%$ increase in the risk of frailty among individuals with low/moderate self-efficacy compared to those with high self-efficacy. Thus, self-efficacy interventions specifically targeted at frailty management may improve the overall health outcomes among stroke survivals.

Although the present study has shown that high selfefficacy is an important factor that can influence or potentially prevent poor frailty outcomes among post-stroke patients, the mechanism underlying our findings may not 
be entirely linear. A possible explanation of the effect of self-efficacy on frailty is through the change in movement behaviour. In the RISE study, individuals with low selfefficacy were found to be less active/sedentary [35]. Previous longitudinal research examined the relationship between frailty and sedentary behaviour in the Toledo Study for Healthy Aging in Spain [36]. The authors indicated that individuals with poor movement behaviour were more likely to have higher frailty scores [36]. It is thus possible that low self-efficacy will influence movement behaviour, which then could affect frailty outcomes. Another potential hypothesis to explain the link between self-efficacy and frailty is the activities of daily living (ADL). ADL has been identified as one of the correlates of frailty in previous studies $[37,38]$. Findings from previous studies have shown that high self-efficacy improve functioning in daily activities $[39,40]$. However, it could be not be determined in the present study if low self-efficacy will reduce ADL functioning. Future studies can investigate if these hypotheses will hold for the relationship between self-efficacy and frailty progression among stroke survivors.

The strength of this study is that it provides evidence to potentially manage the health and wellbeing of stroke survivors. This is because clinical frailty has been suggested to increase the risk of early mortality among poststroke patients [41]. It is thus expected that the knowledge of the predictors of frailty progression among stroke survivors will be vital to foster better post-stroke management strategies. However, the application of the findings from this study should be taken with caution, and the limitations must be acknowledged. One of the limitations of this study is the possible selection bias in the recruitment of the participants. A previous study has indicated that major selection bias occurs in the recruitment of stroke patients into research due to lower mortality among the enrolled participants compared to those not recruited [42]. We have acknowledged that the participants in this study are those with minor strokes. Notwithstanding, the findings highlight the importance of assessing and managing frailty among people living with stroke. The relatively small sample size could also potentially limit the effect sizes and statistical power, which can affect the generalizability of the study findings. We thus recommend that a future study including a larger sample size should deliberately collect more anthropometric data and other frailty-related measurements to assess frailty among people living with stroke. Overall, we believe that the findings from this study added to the knowledge of the modifiable risk factors of frailty and could suggest future interventions for frailty management among stroke survivors.

\section{Conclusion}

In this study, we have examined the frailty trajectory among post-stroke patients and analysed the relationship between frailty and self-efficacy. Our findings showed that low self-efficacy could potentially increase the risk of frailty among stroke survivors. We thus recommend that future studies should explore the use of self-efficacy interventions on frailty management among post-stroke patients.

\section{Acknowledgment}

The corresponding author was hosted at the Fontys University of Applied Sciences, Eindhoven, The Netherlands, as an EarlyStage Researcher on the EuroAgeism secondment programme.

\section{Statement of Ethics}

The Medical Ethics Research Committee approved the RISE study of the University Medical Centre Utrecht (Study No. 14/76). Informed consent was obtained from each of the participants recruited into the RISE study.

\section{Conflict of Interest Statement}

The authors declare that there are no conflicts of interest in this study.

\section{Funding Sources}

This study was funded as part of the EuroAgeism secondment programme. The EuroAgeism project received funding from the European Union's Horizon 2020 research and innovation programme under the Marie Skłodowska-Curie grant agreement No. 764632 .

\section{Author Contributions}

All the authors fulfilled the ICMJE criteria of authorship. A.Q.A. and R.W. designed the study and conducted data analysis. Y.V.Z. and M.P. supervised the data analysis and contributed to manuscript writing and revisions.

\section{Data Availability Statement}

All the data analysed in this study are available upon request. 


\section{References}

1 Johnson CO, Nguyen M, Roth GA, Nichols E, Alam T, Abate D, et al. Global, regional, and national burden of stroke, 1990-2016: a systematic analysis for the global burden of disease study 2016. Lancet Neurol. 2019;18:439_ 58.

2 Avan A, Digaleh H, Di Napoli M, Stranges S, Behrouz R, Shojaeianbabaei G, et al. Socioeconomic status and stroke incidence, prevalence, mortality, and worldwide burden: an ecological analysis from the global burden of disease study 2017. BMC Med. 2019;17:19130.

3 Palmer K, Vetrano DL, Padua L, Romano V, Rivoiro C, Scelfo B, et al. Frailty syndromes in persons with cerebrovascular disease: a systematic review and meta-analysis. Front Neurol. 2019;10:1255

4 Winovich DT, Longstreth WT Jr, Arnold AM, Varadhan R, Zeki Al Hazzouri A, Cushman $\mathrm{M}$, et al. Factors associated with ischemic stroke survival and recovery in older adults. Stroke. 2017;48:1818-26.

5 Rockwood K, Mitnitski A. Frailty in relation to the accumulation of deficits. J Gerontol A Biol Sci Med Sci. 2007;62:722-7.

6 Rockwood K, Howlett SE. Fifteen years of progress in understanding frailty and health in aging. BMC Med. 2018;16:220.

7 Walston JD, Bandeen-Roche K. Frailty: a tale of two concepts. BMC Med. 2015;13:185-3.

8 Rockwood K, Andrew M, Mitnitski A. A comparison of two approaches to measuring frailty in elderly people. J Gerontol A Biol Sci Med Sci. 2007;62:738-43.

9 Dent E, Kowal P, Hoogendijk EO. Frailty measurement in research and clinical practice: a review. Eur J Intern Med. 2016;31:3-10.

10 Myint PK, O Bachmann M, Loke YK, D Musgrave S, Price GM, Hale R, et al. Important factors in predicting mortality outcome from stroke: findings from the anglia stroke clinical network evaluation study. Age Ageing. 2017; 46:83-90.

11 de Sousa Máximo Pereira L, Domingues Dias JM, Guimarães de Assis M. Transition patterns of frailty syndrome in comunity-dwelling elderly individuals: a longitudinal study. J Frail Aging. 2015;4:50-5.

12 Lee JS, Auyeung TW, Leung J, Kwok T, Woo J. Transitions in frailty states among community-living older adults and their associated factors. J Am Med Dir Assoc. 2014;15:281-6.

13 Pollack LR, Litwack-Harrison S, Cawthon PM, Ensrud K, Lane NE, Barrett-Connor E, et al. Patterns and predictors of frailty transitions in older men: the osteoporotic fractures in men study. J Am Geriatr Soc. 2017;65(11): 2473-9.

14 Gardner B, Jovicic A, Belk C, Kharicha K, Iliffe S, Manthorpe J, et al. Specifying the content of home-based health behaviour change interventions for older people with frailty or at risk of frailty: an exploratory systematic review. BMJ Open. 2017;7:e014127.
15 DePew ZS, Karpman C, Novotny PJ, Benzo RP. Correlations between gait speed, 6-minute walk distance, physical activity, and selfefficacy in patients with severe chronic lung disease. Respir Care. 2013;58:2113-9.

16 Fryer CE, Luker JA, McDonnell MN, Hillier SL. Self management programmes for quality of life in people with stroke. Cochrane Database Syst Rev. 2016;22:8.

17 Jones F, Riazi A. Self-efficacy and self-management after stroke: a systematic review. Disabil Rehabil. 2011;33:797.

18 Alharbi M, Gallagher R, Neubeck L, Bauman A, Prebill G, Kirkness A, et al. Exercise barriers and the relationship to self-efficacy for exercise over 12 months of a lifestyle-change program for people with heart disease and/or diabetes. Eur J Cardiovasc Nurs. 2017;16: 309-17.

19 Hladek MD, Gill J, Bandeen-Roche K, Walston J, Allen J, Hinkle JL, et al. High coping selfefficacy associated with lower odds of prefrailty/frailty in older adults with chronic disease. Aging Ment Health. 2020;24:1956-62.

20 Laake K, Laake P, Ranhoff AH, Sveen U, Wyller TB, Bautz-Holter E. The barthel adl index: factor structure depends upon the category of patient (activities of daily living). Age Ageing. 1995;24:393.

21 Rockwood K, Blodgett JM, Theou O, Sun $\mathrm{MH}$, Feridooni HA, Mitnitski A, et al. A frailty index based on deficit accumulation quantifies mortality risk in humans and in mice. Sci Rep. 2017;7:43068.

22 Gahbauer Evelyne A, Mitnitski A, Searle Samuel D, Gill Thomas M, Rockwood K. A standard procedure for creating a frailty index. BMC Geriatrics. 2008;8:24.

23 Cicerone KD, Azulay J. Perceived self-efficacy and life satisfaction after traumatic brain injury; 2007.

24 He Y. Missing data analysis using multiple imputation: getting to the heart of the matter. Circ Cardiovasc Qual Outcomes. 2010;3:98105.

25 Hardin JW. Generalized estimating equations (gee). Encyclopedia of statistics in behavioral science; 2005. Available from:

26 Pisters MF, Veenhof C, Van Dijk GM, Heymans MW, Twisk JW, Dekker J. The course of limitations in activities over 5 years in patients with knee and hip osteoarthritis with moderate functional limitations: risk factors for future functional decline. Osteoarthritis Cartilage. 2012;20:503-10.

27 Han L, Clegg A, Doran T, Fraser L. The impact of frailty on healthcare resource use: a longitudinal analysis using the clinical practice research datalink in england. Age Ageing. 2019;48:665

28 Karunananthan S, Bergman H. Managing frailty in primary care: evidence gaps cannot be ignored. CMAJ. 2018;190:E1122-3

29 Sinclair A. A comparative review of frailty models and a description of the european- wide frailomic initiative: Medical Research Archives; 2018

30 Cesari M, Gambassi G, van Kan GA, Vellas B. The frailty phenotype and the frailty index: different instruments for different purposes. Age Ageing. 2014;43:10-2.

31 Kulminski AM, Ukraintseva SV, Kulminskaya IV, Arbeev KG, Land K, Yashin AI. Cumulative deficits better characterize susceptibility to death in elderly people than phenotypic frailty: lessons from the cardiovascular health study. J Am Geriatr Soc. 2008;56:898-903.

32 Hoogendijk EO, Stenholm S, Ferrucci L, Bandinelli S, Inzitari M, Cesari M. Operationalization of a frailty index among older adults in the inchianti study: predictive ability for allcause and cardiovascular disease mortality. Aging Clin Exp Res. 2020;32:1025-34.

33 Huang HC, Chung KC, Lai DC, Sung SF. The impact of timing and dose of rehabilitation delivery on functional recovery of stroke patients. J Chin Med Assoc. 2009;72:257-64.

34 Torrisi M, De Cola MC, Buda A, Carioti L, Scaltrito MV, Bramanti P, et al. Self-efficacy, poststroke depression, and rehabilitation outcomes: is there a correlation? J Stroke Cerebrovasc Dis. 2018;27:3208-11.

35 Wondergem R, Veenhof C, Wouters E, Bie R, Visser-Meily J, Pisters MF. Movement behavior patterns in people with first-ever stroke; 2019. p. 2020.

36 Mañas A, Pozo-Cruz B, Rodríguez-Gómez I, Losa-Reyna J, Rodríguez-Mañas L, GarcíaGarcía FJ, et al. Which one came first: movement behavior or frailty? A cross-lagged panel model in the toledo study for healthy aging. J Cachexia Sarcopenia Muscle. 2020;11:41523.

37 Vermeulen J, Neyens JC, van Rossum E, Spreeuwenberg MD, de Witte LP. Predicting adl disability in community-dwelling elderly people using physical frailty indicators: a systematic review. BMC Geriatr. 2011;11:33.

38 Gobbens RJJ, van Assen MALM. The prediction of adl and iadl disability using six physical indicators of frailty: a longitudinal study in the Netherlands. Curr Gerontol Geriatr Res. 2014;2014:358137.

39 Kim JH, Park EY. Balance self-efficacy in relation to balance and activities of daily living in community residents with stroke. Disabil Rehabil. 2014;36:295-9.

40 Fridberg H, Gustavsson C. Self-efficacy in activities of daily living and symptom management in people with dizziness: a focus group study. Disabil Rehabil. 2019;41:705-13.

41 Evans NR, Wall J, To B, Wallis SJ, RomeroOrtuno R, Warburton EA. Clinical frailty independently predicts early mortality after ischaemic stroke. Age Ageing. 2020.

$42 \mathrm{Tu}$ JV, Willison DJ, Silver FL, Fang J, Richards JA, Laupacis A, et al. Impracticability of informed consent in the registry of the canadian stroke network. N Engl J Med. 2004;350: 1414-21. 